\title{
BRITISH PAEDIATRIC ASSOCIATION PROCEEDINGS OF THE NINTH ANNUAL GENERAL MEETING
}

The Ninth Annual General Meeting was held at the Old England Lake Hotel, Windermere, on Friday and Saturday, May 8th and 9th, 1936.

\section{First Session (May 8Th, 10 A.m.)}

Business Proceedings: The President, Professor A. E. Naish (Sheffield), was in the Chair, and there were present 52 members.

The Minutes of the last Meeting were read and approved.

The following Officers, Honorary, Corresponding and Ordinary Members were elected :-

President: 1936-37, Dr. Leonard Findlay (London).

Secretary: Dr. A. G. Maitland-Jones (re-elected).

Treasurer: Dr. H. Morley Fletcher (re-elected).

Representative for London: Dr. W. P. H. Sheldon in place of Dr. Alan Moncrieff. Allen.

Representative for Ireland: Dr. R. Marshall (Belfast) in place of Dr. F. M. B.

Honorary Members: Dr. J. H. Thursfield (Past President) and Dr. J. S. Y. Rogers (Dundec).

Corresponding Members: Professor A. Czerny (Germany) and Dr. A. Jeffreys Wood (Australasia).

Ordinary Members: Dr. E. A. Cockayne (London), Dr. H. L. Wallace (Edinburgh) and Dr. T. Pearse Williams (London).

The Treasurer's Report was received and adopted.

It was proposed by Dr. D'Ewart, and seconded by Dr. Morley Fletcher, that a sum of ten guineas be sent to the Royal Medical Benevolent Fund, this being the centenary year of that Association. This was agreed to unanimously.

It was proposed by Dr. Robert Hutchison, and seconded by Dr. Morley Fletcher, that a Sub-Committee of the Association, consisting of Dr. N. Capon and Dr. A. Moncrieff, be set up to consider the standard of weight accepted for the definition of prematurity, this Sub-Committee to have the power to act and to co-opt with the Children's Section of the Royal Society of Medicine. This was agreed to unanimously.

The Secretary then brought to the notice of the Association a letter from the Office of the High Commissioner for India concerning the facilities for PostGraduate Indian Students working for the Diploma of Child Health Examination.

The place of next year's meeting was then discussed, and it was decided to leave the matter in the hands of the Executive Committee. 
1. Dr. Alan Moncrieff (Londen): 'Nasal obstruction in the newborn.' Several examples of this condition, bony or permanent, inflammatory or temporary, were described, and it was emphasised that during the first ten days of life a baby will not naturally breathe through the mouth, even if gross nasal obstruction is present. It was suggested that a soft rubber catheter should be passed through the nostrils in all instances where there appears to be difficulty with breathing.

2. Dr. N. B. CAPON (Liverpool): "Two cases of calcification of the suprarenal glands.' He reported two cases in which the diagnosis of suprarenal calcification was made by radiography. Neither child showed any evidence of tuberculosis, and the clinical histories indicated that in all probability the calcification was secondary to suprarenal haemorrhage during birth. This view seemed particularly probable in case 1, a girl whose age is now sixteen months and who was born by difficult breech delivery; for three days her life was in the balance but she gradually improved. Now she is in a good state of nutrition, but shows spastic diplegia. Case 2 was a girl of thirteen years who died of pneumonia following peritonitis secondary to appendicitis. The notes of her birth and early days of life were not so reliable as in case 1 , but she was delivered by forceps and the labour is said to have been difficult. Unfortunately it was not possible to obtain permission for an autopsy. Brief references were made to the literature, especially to the papers of Baumann, Victor, and Snelling and Erb.

3. Dr. W. Brown (Aberdeen): 'Subnormal temperatures in infancy.' A series of 88 infants, under one year, with rectal temperatures under $98^{\circ} \mathrm{F}$. was described. Of these, 58 were subnormal for long periods (days). Excluding two of the groups, cerebral (15) and prematurity (9), practically all cases in the series showed a history of malnutrition due to vomiting or refusal to feed. Inadequate caloric intake was considered to be the main cause of the low temperature, special proof of this being seen in a pyloric group (16), where the temperature rose immediately following operation or the cessation of spasm. The other 30 cases showed occasional falls, the lowest readings being noted between midnight and 4 a.m. Exposure to cold is a subsidiary factor in causation and was noted often in infants who had been taken a long motor journey in winter. All infants with a temperature below $95^{\circ}$ should have it taken again by a low-recording thermometer : $89^{\circ} \mathrm{F}$. was the lowest recorded temperature in any infant who survived. Treatment consisted of (1) adequate diet with extra glucose, and (2) the application of external heat by means of bottles, electric cage or blanket. For very low readings, below $92^{\circ}$, the cage is used for a short time, but has to be carefully watched; it tends to overheat the infant too quickly and to cause perspiration, resulting in no rise or even a further fall. It is preferable to use a wire cage $7 \frac{1}{2}$ inches righ covered by a thin woollen blanket bearing the electric blanket. A self-recording thermometer is placed heside the infant and read at intervals. A three-way switch regulates the degree of heat. A temperature of over $100^{\circ} \mathrm{F}$. may be reached; thus frequent inspection is necessary to keep it at the optimum of about $90^{\circ} \mathrm{F}$.

4. Dr. R. W. B. Ellis (London): 'Precocious puberty and pseudohermaphroditism.' The relationship and types of these two conditions were discussed with particular reference to six illustrative cases. (1) Interuterine ' interrenalism, in a female infant, the external genitalia being of male type and the internal of female type; the ovary contained true rete testis, and the adrenals were greatly hypertrophied. (2) Pseudohermaphroditism in a female child, with precocious appearance of pubic hair and gigantism. (3) 'Infant Hercules' syndrome in a male infant aged eighteen months. (4) Precocious puberty in a male aged five, and effect of unilateral adrenalectomy. (5) Precocious puberty associated with hydrocephalus and gigantism in a boy aged three and a half. (6) Precocious 
hirsutes and monstrous obesity in a female child of twelve. Reference was also made to the occurrence of precocious puberty in association with tumours of the gonads, of the floor of the third ventricle, and of the pineal body, and to the possible relationship of the two latter to the pituitary. The asymmetric appearance of male characters in the female, and of unilateral gigantism associated with precocious puberty in males, would, in certain instances, imply a chromosomal as well as an endocrine asymmetry.

5. Dr. A. G. Watkins (Cardiff): 'Spontaneous surgical emphysema.' Two cases of spontaneous surgical emphysema were described. The first a girl aged four and a half, following a spasmodic cough-not proven as whooping coughof four days' duration developed surgical emphysema, commencing on the left side of the neck, spreading on to the face and chest wall. This was accompanied by clinical and radiographical signs of a left-sided pneumothorax. Recovery was complete. The second case, a girl aged five, developed extensive surgical emphysema from the scalp to the thighs, first noticed on the left side of the neck. This arose following three days' continuous sneezing, a whooping-cough variant. There were signs of pneumonia at the right base. Although severely ill at the onset, she gradually made a complete recovery. No surgical measures were adopted for the emphysema. A specimen of lung was shown from a boy aged four, who died thirty-six hours after the onset of a severe cough. Associated with a haemorrhagic broncho-pneumonia was interstitial emphysema, interlobar and interlobular, extending towards the hilum. This was taken to illustrate the mechanism by which the subcutaneous emphysema arose, by tracking up the hilar lymphatics and so escaping at the root of the neck.

6. Dr. Hugh T. Asnir (Manchester): "The influence of trauma on the onset of acute poliomyelitis.' A boy aged three and a half years injured his back and fractured the right femur while sliding down a chute in a public park. He was admitted to hospital and ten days later he had a rise of temperature and pain in the right iliac fossa. This was thought to be an attack of mild appendicitis and he was operated upon. The appendix, however, was found to be normal and he soon recovered. When the splints were removed from the leg at the end of a month, the whole leg, from the buttock to the ankle, was found to be paralysed, wasted and cold. He had evidently had an attack of acute infantile paralysis while in hospital and ten days after the accident. Six months later he could only draw the leg up weakly, and he could only walk a few steps without help. It is recorded by several writers that an injury may predispose to or accelerate an attack of infantile paralysis. It was asked, if this is correct, whether the injury in this case had any connection with the infantile paralysis or not.

7. Dr. Leonard) Findely (London): 'Nutritional anaemia in East End of London. He discussed the question of the incidence of nutritional anaemia as shown by examination of the children admitted under his care in the Princess Elizabeth of York Hospital for Children. Contrary to what is frequently stated, he did not find much evidence of anaemia. The haemoglobin and red cell counts showed variations within fairly wide limits, but in only comparatively few instances was the haemoglobin under 70 per cent. (Haldane standard) and the red cell count under 4,000,000 per cent. men. The average haemoglobin and red cell contents, both during infancy and childhood, followed closely the normals of various authors, and particularly closely that described by Dr. R. Hutchison in 1905.

8. Dr. R. C. Jewesbury (London): 'Intra-muscular injection of blood as a therapeutic measure.' The administration of blood is frequently resorted to therapeutically in the treatment of certain conditions, and the mode of introduction of blood is usually by transfusion. In the case of the infant whole blood is given intramuscularly with dramatic success, particularly in cases of haemorrhagic disease 
of the new-born. It is a question as to how far the introduction of whole blood into an infant may be of therapeutic value in conditions other than those which are due to some obvious deficiency in the blood itself. It may be sometimes difficult or even impossible to give a blood transfusion to a wasted, anaemic infant, although special dexterity may be acquired by frequent practice. Furthermore, blood grouping is necessary before the donor's blood can be used. The intramuscular injection of whole blood is an exceedingly simple procedure-20 c.c. can be injected into the buttocks at a time, and it may be repeated at frequent intervals if necessary. The injected blood is rapidly absorbed and produces no untoward reaction. This method has been found of value in the treatment of marasmic infants in whom toxic symptoms have been a marked feature, and the results in this type of case seem to justify further trials.

\section{Skcond Session (May 9Th, 10 a.n.).}

9. Dr. C. P. LAPage (Manchester): 'Micrognathia or hypoplasia of the mandible in the new-born.' Babies with this trouble have a tendency to attacks of cyanosis owing to the tongue falling to the back of the mouth. They are improved by turning on their faces, and may feed better in that position. Their lower jaw shows extreme smallness when attention is focused on it. Cleft palate is common. Treatment was discussed. Extreme care in feeding is necessary at first. Individual attention is important, and much can be done by teaching the baby to push its lower jaw forward with the appliance suggested by Davis and Dunn. The jaw naturally tends to come forward with the development of the lower teeth. A splint which passes round the forehead and tends to push the jaw forwards had been tried in this case without much success. Causation is possibly inter-uterine, perhaps from malposition in utero from pressure of the sternum on the chin. However, strong hereditary tendencies have been found by one author. The importance of the condition is that it may escape detection owing to lack of knowledge of its existence, and much can be done towards permanent recovery, at least in the less severe cases.

10. Dr. G. B. Fleming (Glasgow): 'The blood-sugar in convulsions.' The changes in the level of the blood-sugar in forty children with convulsions were described. The ages of the patients varied from two days to eleven years, and the convulsions were due to various causes:-birth injury, meningitis, spasmophilia, and epilepsy. The blood-sugar during or immediately after the first of a series of convulsions was found in many cases to be raised and gradually fell to low levels. This state of hypoglycaemia lasted for many hours and was not influenced by taking food. It is suggested that the initial hyperglycaemia is due to the cerebral disturbance acting on the suprarenals producing mobilization of glycogen into glucose. The subsequent hypoglycaemia is probably not due to exhaustion of carbohydrate stores in the body, as the ingestion of food containing carbohydrate did not raise the blood-sugar level, but to exhaustion of adrenaline. Against the first hypothesis and in favour of the latter is the observation that, in two cases, injection of adrenaline caused a temporary rise in the blood-sugar level. It is concluded that the disturbance in the carbohydrate metabolism which occurs in convulsions due to various conditions is the result and not the cause of the cerebral disturbance.

11. Dr. C. Wallace Ross (Birmingham), introduced by Prof. L. G. Parsons : 'The carbohydrate metabolism in abdominal tuberculosis.' Two types were described. First, in cases in which the glandular lesions were predominant, flat oral glucose tolerance curves were found to be associated 'with high intravenous ones. Upon this evidence, together with a state of relative insensitivity to injected insulin, the speaker based his belief that there is a defective power to 
absorb carbohydrate in these cases. Secondly, in a case in which ascites was prominent, but without conspicuous glandular enlargement, a different condition was found, a high intravenous result being associated with a high oral one. This was presumed to be due to a toxic state of the patient, of which there was striking clinical evidence. In both types of case the relative intolerance for glucose was presumed to be due to a shortage of 'insulinkinase.' and results were quoted showing that this substance could apparently be supplied, with restoration of tolerance towards normal, either by the injection of campolon intravenously or by the giving of liquid extract of liver by mouth. This had been used in treatment with encouraging results.

12. Dr. N. Morris (Glasgow): "The treatment of diabetes mellitus in childhood.' A summary was given of the experiences at the Diabetic Clinic of the Royal Hospital for Sick Children, Glasgow, during the past ten years. Of fortytwo patients eleven (26.2 per cent.) have died, seven with coma, two with generalized tuberculosis, and two with infectious diseases. No significant difference was noted in the average protein intake per $\mathrm{kgm}$. or insulin per $\mathrm{kgm}$. in the surviving and fatal cases, but in the former the total caloric intake and the percentage of calories as carbohydrate were greater. In the survivors both physical and mental development were good. The effect of increasing the dietary carbohydrate was tested in a group of fourteen patients: of these ten required more total insulin and eight more insulin per $\mathrm{kgm}$. body weight, while six utilized less carbohydrate per unit of insulin. The aims of treatment are to maintain a sense of general well-being and to keep the urine acetone-free and, if possible, sugar-free. The diet is fixed at a level to satisfy each child: a satisfactory first approximation of the dietary requirements is obtained by adding 50 per cent. to the basal figures of Benedict and Talbot. The amount of carbohydrate is left to the child's choice, but a free diet has been found to be unsatisfactory. In some patients seasonal variations in insulin requirements were noted, less being necessary in the summer months.

13. Dr. B. Schlesinger (London): ' Treatment of enteritis by apple diet.' After describing the method of preparing the apples, the mode of administration and the transitional diet which it was necessary for the child to have before resuming his normal meals, he discussed the type of case in which most benefit was obtained. Infants under one year were not generally suitable, but the treatment appeared to be equally $y_{i}$ effective in all acute types of infection. Some of his cases had been severe examples of bacillary dysentery with fever, great shock, vomiting and innumerable stools containing large quantities of blood and mucus. The patients were thoroughly ill and seemed to recover more rapidly with apple diet than other similar cases had in his experience with the more usual methods of treatment. There was no guarantee, however, against the continued presence of dysentery bacilli in the stools, although the patients themselves seemed to have recovered.

14. Dr. G. H. Newns and Dr. R. Wilson (London), introduced by Dr. D. Paterson: 'The treatment of pyelitis in children by mandelic acid.' Thirty-five cases treated with mandelic acid at the Hospital for Sick Children, Great Ormond Street, and Westminster Hospital were reported. The majority were girls over two years. In thirty cases the infection was due to B.coli. Five cases showed evidence of kidney damage or renal tract abnormality, the remaining having apparently straightforward pyelitis. Mandelic acid (with sodium bicarbonate) was used in most cases. The dose varied from 60 to 120 grains daily according to the age of the child. Ammonium chloride, in doses of about half the above, was given to make the urine acid. Neocit (a powdered preparation of mandelic acid and sodium bicarbonate) was used in six cases. This obviated the necessity of 
giving ammonium chloride. This preparation did not, however, maintain the $\mathrm{pH}$ of the urine constantly at the necessary low level. A pH of 5.6 is necessary for the maximum bacteriocidal action of the mandelic acid to be obtained. No toxic effects on the kidney were observed in any of the cases. In thirty-two cases, the urine was rendered sterile, in many within a few days. This result was obtained in nine cases in which potassium citrate had been previously given without effect. The patients had urinary examinations periodically after discharge. Ten cases were found to have relapsed. Half of these may be ascribed to renal abnormalities or a non-B.coli infection. Mandelic acid appears to be an effective agent for rendering the urine sterile in cases of pyelitis. The tendency to recurrence might be minimized by more prolonged treatment.

15. Dr. J. H. Ebrs (Birmingham), introduced by Prof. L. G. Parsons : 'A clinical syndome associated with cerebral sinus thrombosis.' Twenty-one cases of cerebral sinus thrombosis were reviewed briefly and two cases described in detail, both females, aged sixteen months and two years respectively, the outstanding points being a sudden onset with restlessness, followed by convulsions; localization of the convulsions as shown by twitching and followed by paresis; marked and persistent drowsiness; the cerebro-spinal fluid findings of increased pressure, normal number of cells, cultures sterile, low sugar content, chloride values of $774 \mathrm{mgm}$. per cent. in one case, and $932 \mathrm{mgm}$. per cent. in the other, raised protein; the absence of any clinical evidence of infection; and at post mortem, thrombosis of the cerebral vessels. The chloride value of $932 \mathrm{mgm}$. per cent. in the cerebro-spinal fluid is the highest value in the records of the Birmingham Children's Hospital, and has not yet been explained. The cases represent two unusual examples of cerebral sinus thrombosis in children which have probably resulted from some remote infection. 